\title{
Usefulness of four-limb blood pressure measurement in prediction of overall and cardiovascular mortality in acute myocardial infarction
}

\author{
Po-Chao Hsu'1,3, Wen-Hsien Lee ${ }^{1,2,3}$, Wei-Chung Tsai ${ }^{1,3}$, Chun-Yuan Chu1 ${ }^{1}$, Chee-Siong Lee ${ }^{1,3}$, Hsueh-Wei \\ Yen ${ }^{1,3}$, Tsung-Hsien Lin 1,3, Wen-Chol Voon 1,3, Wen-Ter Lai ${ }^{1,3}$, Sheng-Hsiung Sheu1,3, Ho-Ming Su ${ }^{1,2,3}{ }^{\circledR}$ \\ 1. Division of Cardiology, Department of Internal Medicine, Kaohsiung Medical University Hospital, Kaohsiung, Taiwan. \\ 2. Department of Internal Medicine, Kaohsiung Municipal Siaogang Hospital, Kaohsiung, Taiwan. \\ 3. Faculty of Medicine, College of Medicine, Kaohsiung Medical University, Kaohsiung, Taiwan. \\ $\square$ Corresponding author: Ho-Ming Su, MD, Department of Internal Medicine, Kaohsiung Municipal Siaogang Hospital, Kaohsiung Medical University, 482 \\ Shan-Ming Rd., Hsiao-Kang Dist., 812 Kaohsiung, Taiwan, R.O.C. Tel: 886- 7- 8036783 - 3441, Fax: 886- 7- 8063346, E-mail: cobeshm@seed.net.tw. \\ ( ) The author(s). This is an open access article distributed under the terms of the Creative Commons Attribution License (https://creativecommons.org/licenses/by/4.0/). \\ See http://ivyspring.com/terms for full terms and conditions.
}

Received: 2020.02.09; Accepted: 2020.05.12; Published: 2020.05.23

\begin{abstract}
Four-limb blood pressure measurement could improve mortality prediction in the elderly. However, there was no study to evaluate whether such measurement was still useful in predicting overall and cardiovascular (CV) mortality in acute myocardial infarction (AMI). Two hundred AMI patients admitted to cardiac care unit were enrolled. The 4-limb blood pressures, inter-limb blood pressure differences,

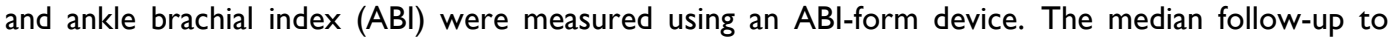
mortality was 64 months (25th-75th percentile: 5-174 months). There were 40 and 138 patients documented as $C V$ and overall mortality, respectively. After multivariable adjustment, the ankle diastolic blood pressure (DBP) on the lower side, $A B I$ value, $A B I<0.9$, interarm $D B P$ difference, interankle systolic blood pressure (SBP) and DBP differences, interankle SBP difference $\geq 15 \mathrm{mmHg}$, and interankle DBP difference $\geq 10 \mathrm{mmHg}$ could predict overall mortality $(P \leq 0.025)$. The ankle DBP on the lower side, interankle DBP difference, and interankle DBP difference $\geq 10 \mathrm{mmHg}$ could predict $\mathrm{CV}$ mortality $(\mathrm{P} \leq$ 0.031). In addition, in the Nested Cox model, the model including the ankle DBP on the lower side and the model including interankle DBP difference had the best value for overall and CV mortality prediction, respectively $(P \leq 0.031)$. In AMl patients, 4-limb blood pressure measurement could generate several useful parameters in predicting overall and CV mortality. Furthermore, ankle DBP on the lower side and interankle DBP difference were the most powerful parameters in prediction of overall and CV mortality, respectively.
\end{abstract}

Key words: 4-limb blood pressure; interarm blood pressure difference; interankle blood pressure difference; ankle-brachial index; acute myocardial infarction

\section{Introduction}

Current technology makes simultaneous blood pressure measurement in 4 limbs easy [1], which can provide a complete evaluation of blood pressures and generate a reliable value of blood pressure differences among 4 limbs. An interarm systolic blood pressure (SBP) difference $\geq 10 \mathrm{mmHg}$ was reported to be significantly associated to peripheral vascular disease, coronary artery disease, and increased cardiovascular (CV) and overall mortality [2-5]. Besides, an interankle SBP difference $\geq 15 \mathrm{mmHg}$ was also demonstrated to be correlated to increased $\mathrm{CV}$ and overall mortality in patients with hemodialysis [6] and in elderly Chinese population [5].

The ankle-brachial index (ABI) is a simple and noninvasive method to diagnose peripheral artery disease and an $\mathrm{ABI}<0.9$ has been frequently used to confirm the diagnosis of peripheral artery occlusive disease [7]. Additionally, a low ABI was found to be able to predict $\mathrm{CV}$ and overall mortality [8-10]. In a large national screening database, there is a strong 
and consistent relationship between ABI level and a history of prevalent myocardial infarction [11]. Compared to patients without peripheral artery disease, acute myocardial infarction (AMI) patients with peripheral artery disease were older, had more comorbidities, and had a higher rate of adverse $\mathrm{CV}$ events [12].

Sheng et al. found above and beyond arm blood pressure level, the interarm and interankle blood pressure differences and ABI derived from simultaneous 4-limb blood pressure measurement could improve the prediction of mortality in the elderly [5]. However, there was no study to evaluate whether simultaneous 4-limb blood pressure measurement was still useful in prediction of $\mathrm{CV}$ and overall mortality in AMI patients, a group of patients with high prevalence of peripheral artery disease [13]. Hence, the present study was designed to examine the ability of 4-limb blood pressures, inter-limb blood pressure differences, and $\mathrm{ABI}$ in prediction of $\mathrm{CV}$ and overall mortality in AMI patients. In addition, we also compare the prediction values for $\mathrm{CV}$ and overall mortality among these parameters in such patients.

\section{Materials and Methods}

\section{Study population and design}

This observational cohort study consecutively included AMI (ST segment elevation AMI and non-ST segment elevation AMI) patients admitted to our cardiac care unit from November 2003 to September 2004. Patients with atrial fibrillation and limb amputation were excluded. Finally, 200 AMI patients were included in this study. CV and overall mortality data were collected up to December 2018. Mortality data were obtained from the Collaboration Center of Health Information Application (CCHIA), Ministry of Health and Welfare, Executive Yuan, Taiwan.

\section{Ethics Statement}

The study protocol was approved by the institutional review board (IRB) committee of our Hospital. Informed consents have obtained in written form from patients and all clinical investigation was conducted according to the principles expressed in the Declaration of Helsinki.

\section{Assessment of 4-limb blood pressures, inter-limb blood pressure differences, and ABI}

The values of four-limb SBPs, diastolic blood pressures (DBPs), interarm and interankle SBP and DBP differences, and ABI were measured by using an ABI-form device (VP1000; Colin Co. Ltd., Komaki, Japan), which automatically and simultaneously measured blood pressures in both arms and ankles using an oscillometric method [14,15]. The ABI-form device measurement was done once in each patient and was performed within 24 hours of admission to cardiac care unit. Interarm blood pressure differences were derived from the SBP and DBP differences between right and left arms. Interankle blood pressure differences were derived from the SBP and DBP differences between right and left ankles. The ABI was calculated by the ratio of the ankle SBP divided by the higher SBP of the arms. After obtaining bilateral $\mathrm{ABI}$ values, the lower one was used for later analysis.

\section{Collection of demographic and medical data}

Demographic and medical data including age, gender, body mass index, and comorbid conditions such as diabetes and hypertension were obtained from medical records.

\section{Definition of overall and CV mortality}

All study participants were followed up till December 2018. Survival information and causes of death were obtained from the official death certificate and final confirmation by the Ministry of Health and Welfare. The causes of death were classified by the International Classification of Diseases 10th Revision. Causes of CV mortality were defined deaths due to hypertensive disease, cardiac disease, cerebral vascular disease, ischemic heart disease, myocardial infarction, heart failure, valvular heart disease, and atherosclerotic vascular disease. No participant lost follow-up in our study.

\section{Statistical analysis}

All statistical analyses were performed with SPSS 22.0 software (SPSS, Chicago, IL, USA). Data were expressed as mean \pm standard deviation, percentage, or median (25th-75th percentile) for follow-up period. Continuous and categorical variables between groups were compared by independent samples t-test and Chi-square test, respectively. Time to the $\mathrm{CV}$ and overall mortality and covariates of risk factors were adjusted using a Cox proportional hazards model. A significant improvement in model prediction was based on the chi-square statistic, which followed a difference in chi-square value and the $\mathrm{P}$ value was based on the value compared with the basic model. Kaplan-Meier survival plots were calculated from baseline to time of mortality events. All tests were 2 -sided and $\mathrm{P}$ values less than 0.05 were considered statistically significant.

\section{Results}

Among the 200 subjects, mean age was $66.2 \pm$ 13.6 years. There were 40 and 160 patients with ST segment elevation AMI and non-ST segment elevation 
AMI, respectively. The prevalence of interarm SBP difference $\geq 10 \mathrm{mmHg}$, interarm DBP difference $\geq 10$ $\mathrm{mmHg}$, interankle SBP difference $\geq 15 \mathrm{mmHg}$, and interankle DBP difference $\geq 10 \mathrm{mmHg}$ were $14.4 \%$, $5.3 \%, 33.3 \%$, and $17.7 \%$, respectively.

Table 1 compares the clinical characteristics between patients with and without mortality. Compared to patients without mortality, patients with mortality were found to have an older age, less male sex, lower BMI, higher prevalence of hypertension, higher left and right arm SBPs, lower left and right ankle DBPs, lower ABI value, higher prevalence of $\mathrm{ABI}<0.9$, higher interarm $\mathrm{DBP}$ difference, higher interankle SBP and DBP differences, and higher prevalence of interankle SBP difference $\geq 15 \mathrm{mmHg}$ and interankle DBP difference $\geq$ $10 \mathrm{mmHg}$. There were no significant differences for dyslipidemia and smoking $(\mathrm{P}=0.834$ and $\mathrm{P}=0.942$, respectively).

The median follow-up to mortality was 64 months (25th-75th percentile: 5-174 months) in all patients. Mortality events were documented during the follow-up period, including CV mortality $(n=40)$ and overall mortality $(n=138)$. For in-hospital mortality, there were 8 patients with $\mathrm{CV}$ mortality and 30 patients with overall mortality.

Table 1. Baseline characteristics of the study population by mortality

\begin{tabular}{|c|c|c|c|c|}
\hline Baseline Characteristics & Mortality (+) & Mortality (-) & $P$ value & Total patients \\
\hline Number & 138 & 62 & & 200 \\
\hline Age (yr) & $71 \pm 11$ & $55 \pm 12$ & $<0.001$ & $66 \pm 14$ \\
\hline Male gender (\%) & $65.9 \%$ & $83.9 \%$ & 0.011 & $71.5 \%$ \\
\hline $\mathrm{DM}(\%)$ & $29.7 \%$ & $25.8 \%$ & 0.615 & $28.5 \%$ \\
\hline $\mathrm{H} / \mathrm{T}(\%)$ & $47.1 \%$ & $27.4 \%$ & 0.013 & $41.0 \%$ \\
\hline Dyslipidemia (\%) & $31.2 \%$ & $30.6 \%$ & 0.942 & $31.0 \%$ \\
\hline Smoking (\%) & $52.6 \%$ & $50.0 \%$ & 0.834 & $51.9 \%$ \\
\hline $\operatorname{BMI}\left(\mathrm{kg} / \mathrm{m}^{2}\right)$ & $23.5 \pm 3.9$ & $25.5 \pm 3.2$ & $<0.001$ & $24.1 \pm 3.8$ \\
\hline \multicolumn{5}{|l|}{ Simultaneous 4-limb BP } \\
\hline Left arm SBP (mmHg) & $126 \pm 21$ & $117 \pm 17$ & 0.002 & $123 \pm 20$ \\
\hline Left arm DBP (mmHg) & $71 \pm 12$ & $72 \pm 12$ & 0.647 & $71 \pm 12$ \\
\hline Right arm SBP (mmHg) & $128 \pm 23$ & $118 \pm 18$ & 0.002 & $125 \pm 22$ \\
\hline Right arm DBP (mmHg) & $72 \pm 13$ & $73 \pm 13$ & 0.595 & $72 \pm 13$ \\
\hline Left ankle SBP (mmHg) & $124 \pm 37$ & $126 \pm 22$ & 0.547 & $124 \pm 33$ \\
\hline Left ankle DBP (mmHg) & $66 \pm 19$ & $73 \pm 12$ & 0.003 & $68 \pm 17$ \\
\hline Right ankle SBP (mmHg) & $126 \pm 35$ & $129 \pm 23$ & 0.525 & $127 \pm 31$ \\
\hline Right ankle DBP (mmHg) & $65 \pm 19$ & $73 \pm 12$ & 0.001 & $68 \pm 18$ \\
\hline \multicolumn{5}{|l|}{ ABI data } \\
\hline ABI value & $0.90 \pm 0.23$ & $1.03 \pm 0.11$ & $<0.001$ & $0.94 \pm 0.21$ \\
\hline$<0.9(\%)$ & $38.4 \%$ & $6.5 \%$ & $<0.001$ & $28.5 \%$ \\
\hline \multicolumn{5}{|l|}{ Interarm BP difference } \\
\hline $\mathrm{SBP}(\mathrm{mmHg})$ & $5.9 \pm 5.2$ & $5.0 \pm 3.9$ & 0.254 & $5.6 \pm 4.8$ \\
\hline $\mathrm{DBP}(\mathrm{mmHg})$ & $5.1 \pm 4.4$ & $3.6 \pm 3.0$ & 0.023 & $4.6 \pm 4.0$ \\
\hline $\mathrm{SBP} \geq 10 \mathrm{mmHg}(\%)$ & $15.9 \%$ & $11.3 \%$ & 0.509 & $14.4 \%$ \\
\hline $\mathrm{DBP} \geq 10 \mathrm{mmHg}(\%)$ & $7.2 \%$ & $1.6 \%$ & 0.169 & $5.3 \%$ \\
\hline \multicolumn{5}{|l|}{ Interankle BP difference } \\
\hline $\mathrm{SBP}(\mathrm{mmHg})$ & $17.9 \pm 20.0$ & $7.2 \pm 6.2$ & $<0.001$ & $14.4 \pm 17.3$ \\
\hline $\mathrm{DBP}(\mathrm{mmHg})$ & $8.4 \pm 10.0$ & $3.4 \pm 2.8$ & $<0.001$ & $6.7 \pm 8.7$ \\
\hline $\mathrm{SBP} \geq 15 \mathrm{mmHg}(\%)$ & $43.3 \%$ & $12.9 \%$ & $<0.001$ & $33.3 \%$ \\
\hline $\mathrm{DBP} \geq 10 \mathrm{mmHg}(\%)$ & $25.8 \%$ & $1.6 \%$ & $<0.001$ & $17.7 \%$ \\
\hline
\end{tabular}

Abbreviations: $\mathrm{ABI}$, ankle-brachial index; BMI, body mass index; $\mathrm{BP}$, blood pressure; DBP, diastolic blood pressure; $\mathrm{DM}$, diabetes mellitus; $\mathrm{H} / \mathrm{T}$, hypertension; SBP, systolic blood pressure.
Table 2 shows the predictors of overall and CV mortality using Cox proportional hazards model in the multivariable analysis after adjustment for age, sex, body mass index, diabetes, and arm SBP on the higher side. If we just adjusted above parameters as basic model, age is the only significant predictor for long-term overall and CV mortality $(\mathrm{P}<0.001$ and $\mathrm{P}=$ 0.007 , respectively). Then we further added other variables in to the basic model. For blood pressures of lower limb, the ankle DBP on the lower side could predict long-term overall and CV mortality. For ABI data, ABI value itself and ABI $<0.9$ could predict overall mortality, but could not predict CV mortality. For interarm blood pressure differences, only DBP difference could predict overall mortality. For interankle blood pressure differences, all of SBP difference, DBP difference, SBP difference $\geq 15 \mathrm{mmHg}$, and DBP difference $\geq 10 \mathrm{mmHg}$ could predict overall mortality. However, only DBP difference and DBP difference $\geq 10 \mathrm{mmHg}$ could predict $\mathrm{CV}$ mortality in our study.

Table 2. Predictors of overall and cardiovascular mortality using Cox proportional hazards model

\begin{tabular}{|c|c|c|c|c|}
\hline \multirow[t]{2}{*}{ Parameter } & \multicolumn{2}{|l|}{ Overall mortality } & \multicolumn{2}{|l|}{$\begin{array}{l}\text { Cardiovascular } \\
\text { mortality }\end{array}$} \\
\hline & HR (95\% CI) & $P$ & $\mathrm{HR}(95 \% \mathrm{CI})$ & $P$ \\
\hline \multicolumn{5}{|l|}{ BP of lower limb } \\
\hline Ankle SBP on the higher side & $0.998(0.991-1.005)$ & 0.563 & $1.000(0.987-1.014)$ & 0.954 \\
\hline Ankle DBP on the lower side & $0.980(0.969-0.991)$ & $<0.001$ & $0.977(0.957-0.998)$ & 0.031 \\
\hline \multicolumn{5}{|c|}{ ABI data } \\
\hline ABI value & $0.380(0.162-0.887)$ & 0.025 & $0.516(0.102-2.603)$ & 0.423 \\
\hline$<0.9$ & $1.917(1.286-2.858)$ & 0.001 & $2.061(0.982-4.328)$ & 0.056 \\
\hline \multicolumn{5}{|l|}{ Interarm BP difference } \\
\hline SBP & $1.006(0.970-1.044)$ & 0.746 & $1.029(0.963-1.100)$ & 0.395 \\
\hline DBP & 1.052(1.007-1.100) & 0.023 & $1.045(0.962-1.135)$ & 0.300 \\
\hline $\mathrm{SBP} \geq 10 \mathrm{mmHg}$ & $1.209(0.730-2.002)$ & 0.461 & $1.957(0.851-4.503)$ & 0.114 \\
\hline $\mathrm{DBP} \geq 10 \mathrm{mmHg}$ & $1.991(0.978-4.056)$ & 0.058 & $1.591(0.371-6.829)$ & 0.532 \\
\hline \multicolumn{5}{|l|}{ Interankle BP difference } \\
\hline SBP & $1.019(1.009-1.029)$ & $<0.001$ & $1.011(0.993-1.030)$ & 0.228 \\
\hline $\mathrm{DBP}$ & $1.030(1.010-1.050)$ & 0.002 & $1.040(1.010-1.071)$ & 0.009 \\
\hline $\mathrm{SBP} \geq 15 \mathrm{mmHg}$ & $1.809(1.232-2.655)$ & 0.002 & $0.923(0.444-1.920)$ & 0.831 \\
\hline $\mathrm{DBP} \geq 10 \mathrm{mmHg}$ & $2.087(1.275-3.416)$ & 0.003 & $2.572(1.093-6.051)$ & 0.030 \\
\hline
\end{tabular}

Figure 1A shows the Nested Cox model for overall mortality. The basic model included age, sex, body mass index, diabetes, and arm SBP on the higher side. The basic model could significantly predict overall mortality (Chi-square vale, $84.7, \mathrm{P}<0.001$ ). We added the significant parameters in the Table 2, including ankle $\mathrm{DBP}$ on the lower side, $\mathrm{ABI}$ value, $\mathrm{ABI}<0.9$, interarm DBP difference, interankle SBP and DBP differences, and interankle SBP difference $\geq$ $15 \mathrm{mmHg}$ and DBP difference $\geq 10 \mathrm{mmHg}$, into the basic model one by one. After comparing the Chisquare values, the model including the ankle DBP on 
the lower side had the best predictive value for overall mortality. In addition, the model consisting of interarm DBP difference had the lowest prediction value for overall mortality.

Figure 1B shows the Nested Cox model for CV mortality. The basic model included age, sex, body mass index, diabetes, and arm SBP on the higher side. The basic model could significantly predict CV mortality (Chi-square vale, $16.3, \mathrm{P}=0.006$ ). We added the significant parameters in the Table 2, including ankle DBP on the lower side, interankle DBP difference, and interankle DBP difference $\geq 10 \mathrm{mmHg}$, into the basic mode one by one. After comparing the Chi-square values, the model including interankle DBP difference had the highest predictive valve for CV mortality.

Figure 2 illustrates the Kaplan-Meier curves for adjusted overall mortality-free survival (Figure 2A: ABI $<0.9$ versus $\geq 0.9$; Figure 2B: interankle SBP difference $\geq 15 \mathrm{mmHg}$ versus $<15 \mathrm{mmHg}$; Figure $2 \mathrm{C}$ : interankle DBP difference $\geq 10 \mathrm{mmHg}$ versus < $10 \mathrm{mmHg}$ ). Figure 3 illustrates the Kaplan-Meier curve for adjusted CV mortality-free survival (interankle DBP difference $\geq 10 \mathrm{mmHg}$ versus $<10 \mathrm{mmHg}$ ).

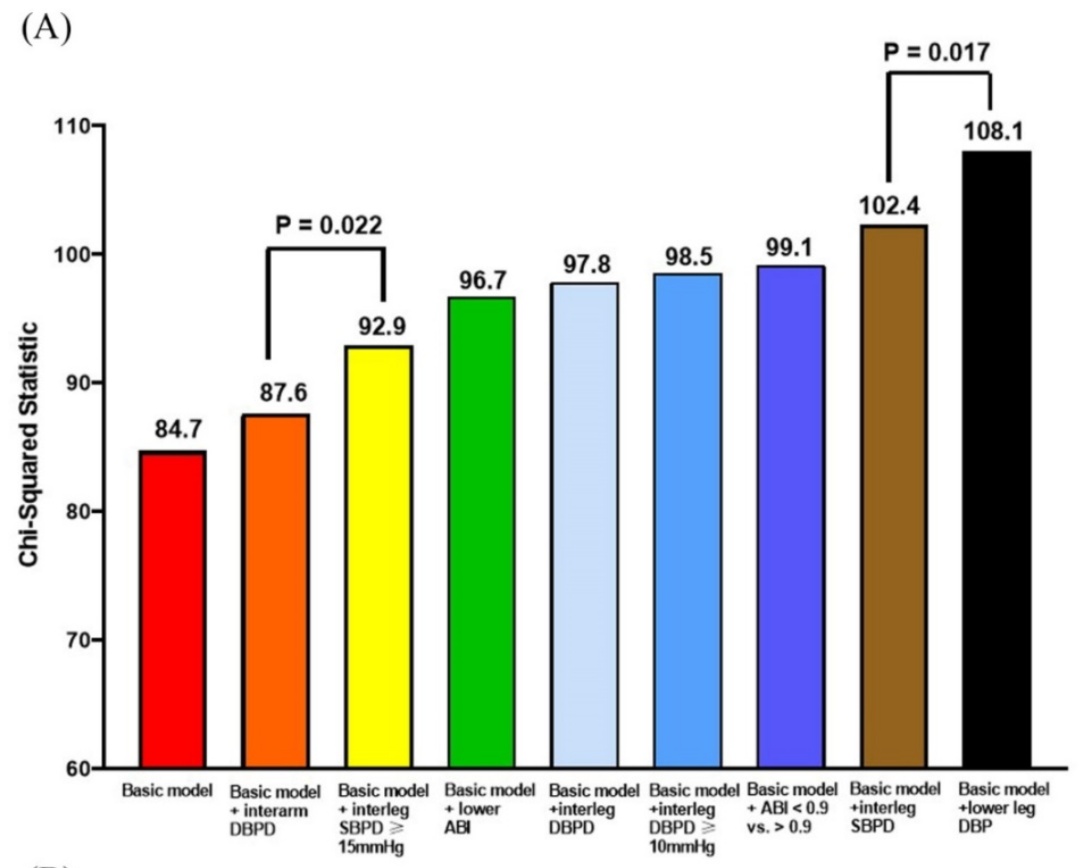

(B)

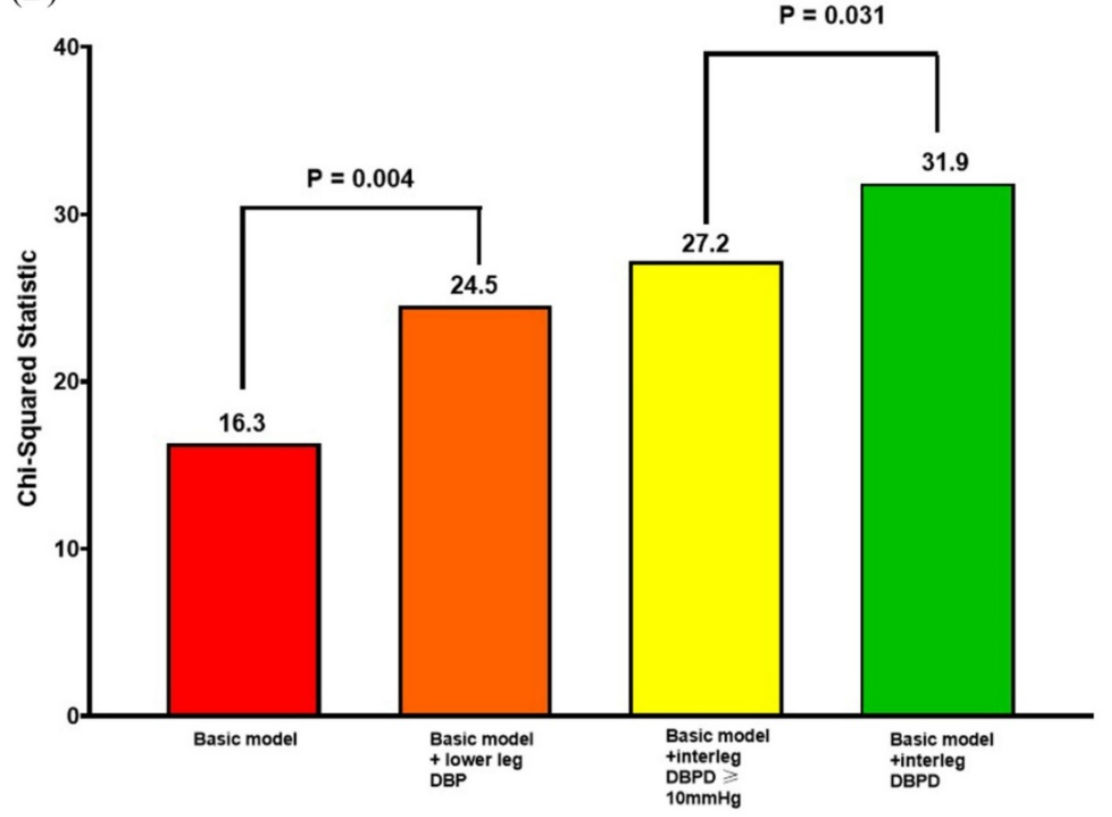

Figure 1. Nested Cox model for overall mortality and CV mortality. (A) Nested Cox model for overall mortality; (B) nested Cox model for CV mortality. Basic model: Adjustment for age, sex, BMI, diabetes, and arm SBP on the higher side. Abbreviations: ABI, ankle-brachial index; BMI: body mass index; CV: cardiovascular; DBP: diastolic blood pressure; DBPD: diastolic blood pressure difference; SBP: systolic blood pressure; SBPD: systolic blood pressure difference. 

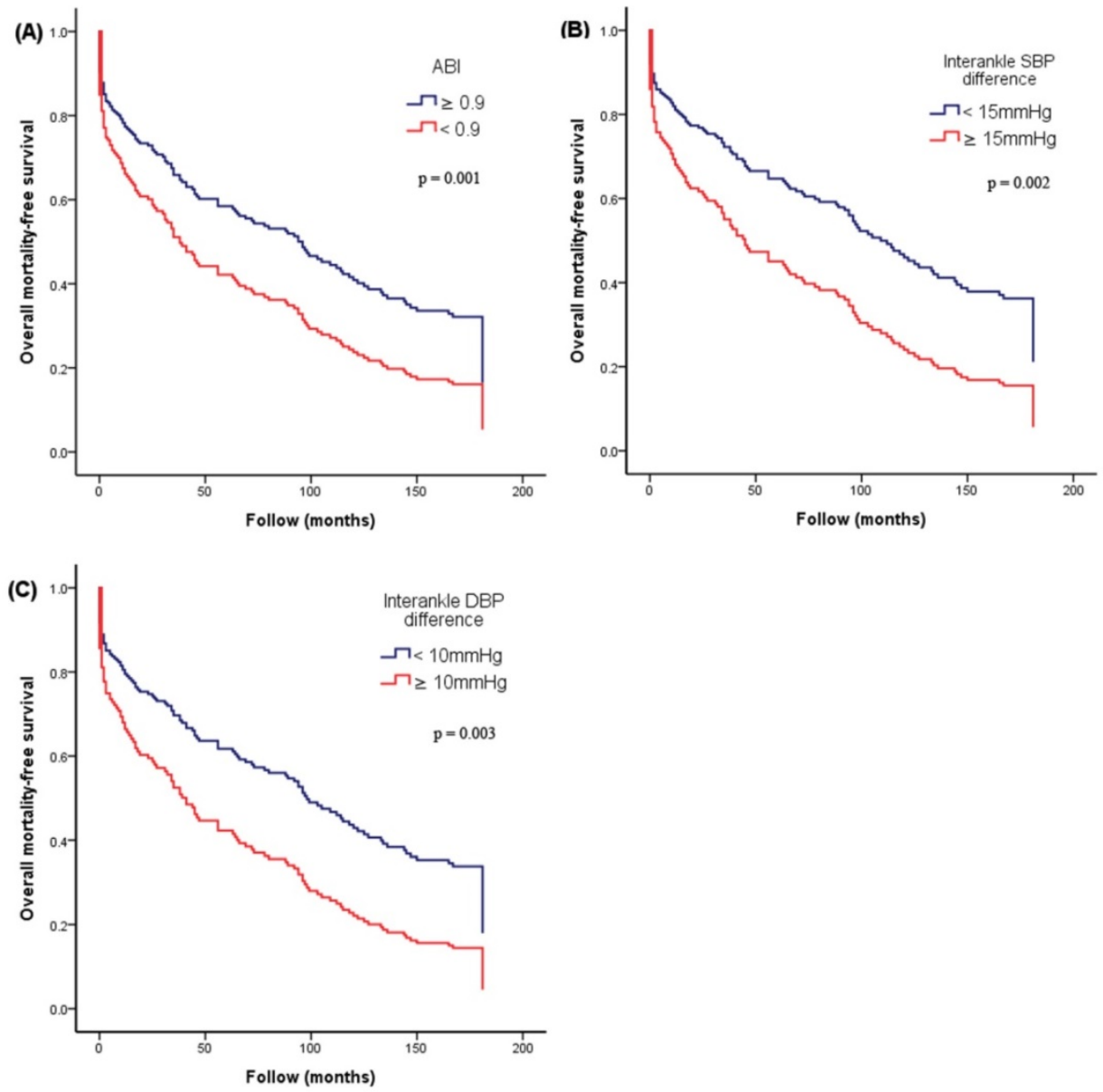

Figure 2. Kaplan-Meier curves for adjusted overall mortality-free survival. (A) $A B I<0.9$ versus $\geq 0.9$; (B) interankle $S B P$ difference $\geq 15 \mathrm{mmHg}$ versus $<15 \mathrm{mmHg}$; (C) interankle DBP difference $\geq 10 \mathrm{mmHg}$ versus < $10 \mathrm{mmHg}$ ). Adjustment for age, sex, BMl, diabetes, and arm SBP on the higher side. Abbreviations: ABI, ankle-brachial index; BMI, body mass index; DBP, diastolic blood pressure; SBP, systolic blood pressure.

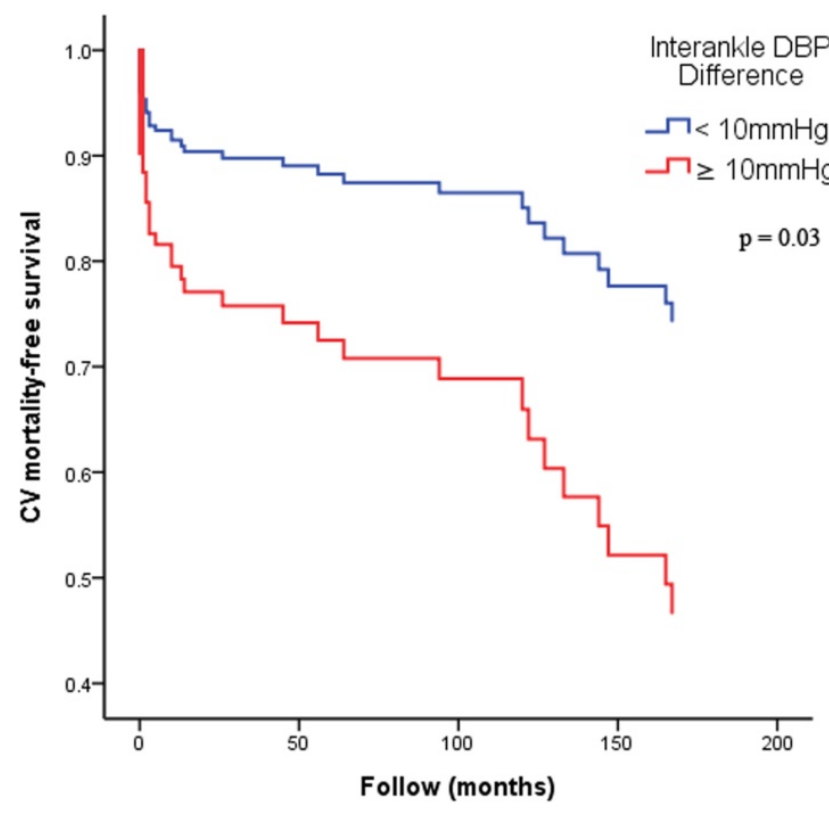

Figure 3. Kaplan-Meier curves for adjusted CV mortality-free survival. Adjustment for age, sex, BMI, diabetes, and arm SBP on the higher side. Abbreviations: BMI, body mass index; CV, cardiovascular; DBP, diastolic blood pressure; SBP, systolic blood pressure.

\section{Discussion}

This study aimed to evaluate the impact of 4-limb blood pressure measurement on the prediction of overall and CV mortality in AMI patients. We found ankle DBP on the lower side, ABI value, ABI < 0.9 , interarm DBP difference, interankle SBP and DBP differences, interankle SBP difference $\geq 15 \mathrm{mmHg}$, and interankle DBP difference $\geq 10 \mathrm{mmHg}$ could predict long term overall mortality. In addition, the ankle DBP on the lower side, interankle DBP difference, and interankle DBP difference $\geq 10 \mathrm{mmHg}$ could predict long term CV mortality. Among these 8 parameters for prediction of overall mortality, 7 parameters could not be obtained without lower limb measurement and 4 parameters belonged to DBP itself or its derived parameters. Among these 3 parameters for prediction of CV mortality, all of them could not be obtained without lower limb blood pressure measurement and all of them belonged to ankle DBP itself or its derived parameters. Hence, for better mortality prediction in AMI patients, simultaneous 4-limb blood pressure measurement was necessary 
and really had a big impact on the survival prediction. The easily-overlooked ankle DBP and its derived parameters should be taken into consideration.

Simultaneous measurement of blood pressures is preferred in assessment of SBP and DBP differences between limbs because they can avoid overestimation of interarm and interankle blood pressure differences, which may be caused by short-term blood pressure variability or white coat effects $[16,17]$. Therefore, the ABI-form device (VP1000; Colin Co. Ltd., Komaki, Japan) used in this study was a very suitable tool to evaluate the values of interarm and interankle blood pressure difference $[14,15]$.

Interarm blood pressure difference was not an uncommon phenomenon. Typically, interarm SBP difference $\geq 10 \mathrm{mmHg}$ was found in $4.4 \%$ subjects free of vascular disease. However, the prevalence increased to $7 \%$ in diabetic and $13.6 \%$ in hypertensive patients [18]. In our study, patients with AMI had $14.4 \%$ cases with interarm SBP difference $\geq 10 \mathrm{mmHg}$, which was similar with the previous study with hypertensive patients. Interarm SBP difference was associated with $\mathrm{CV}$ and overall mortality in various populations, such as patients with hypertension, diabetes, cerebrovascular disease, coronary artery disease, and so on [4,19-23]. In a meta-analysis, an interarm SBP difference of $\geq 15 \mathrm{mmHg}$ was associated with a $55 \%$ and $68 \%$ increase in overall and CV mortality, respectively [22]. Not similar with the previous findings, the present study demonstrated that both of the value of interarm SBP difference and interarm SBP difference $\geq 10 \mathrm{mmHg}$ had no association with overall and CV mortality in AMI patients. However, the value of interarm DBP difference could predict overall mortality in the multivariable analysis in our study. DBP was one of the major determinants of coronary perfusion pressure [24,25]. Chang et al. ever showed interarm DBP difference $\geq 10 \mathrm{mmHg}$ was related to early neurological deterioration, poor functional outcome, and mortality in stroke patients [26]. Hence, interarm DBP difference might be more useful than interarm SBP difference in prediction of overall mortality in AMI patients.

Our previous study showed interankle SBP difference $\geq 15 \mathrm{mmHg}$ was independently associated with $\mathrm{ABI}<0.9$, higher brachial-ankle pulse wave velocity, and increased overall and CV mortality in patients with hemodialysis [6]. We also evaluated interankle SBP difference in stage 3-5 chronic kidney disease patients and found interankle SBP difference was associated with rapid progression and progression to renal end points [27]. Sheng et al. also found not only increased interarm SBP and DBP differences, but also increased interankle SBP and
DBP differences were associated with $\mathrm{CV}$ and overall mortality in the Chinese elderly after 4 years followup [5]. Therefore, interankle blood pressure differences should not be ignored when performing survival analysis. In this study, as shown in Table 2, all of four parameters of interankle blood pressure differences and two parameters of interankle DBP differences could predict overall and CV mortality, respectively. Hence, calculation of interankle blood pressure differences, especially the DBP difference, might be very helpful in mortality prediction in patients with AMI.

In our study, in addition to the value of interankle DBP difference and interankle DBP difference $\geq 10 \mathrm{mmHg}$, the ankle DBP on the lower side also could predict overall and CV mortality. Furthermore, in the Nested Cox model, the addition of ankle DBP on the lower side into the basic model had the highest valve in predicting overall mortality. Hence, our results suggested that both of ankle DBP itself and its derived parameters played an important role in overall and CV morality prediction in AMI. Generally speaking, it was not difficult to understand the importance of DBP in patients with AMI. DBP had a J-curve relationship with coronary artery disease and death. Such association was thought to reflect reduced coronary perfusion at low DBP [24,25]. Rahman et al. reported that DBP $<60 \mathrm{mmHg}$ was associated with increased risk of coronary events and all-cause mortality [24]. Protogerou et al. also showed DBP $\leq 60 \mathrm{mmHg}$ was associated with reduced survival in the frail elderly [25]. These results supported our present finding, i.e. a significant association of DBP with overall and $\mathrm{CV}$ mortality. Hence, additional consideration of ankle DBP itself and its derived parameters might provide extra benefit in prediction of overall and CV mortality in AMI patients.

A low ABI was reported to be associated with an increased $\mathrm{CV}$ and overall mortality in different population, such as patients with chronic kidney disease [28], hemodialysis [29], diabetes [30], and hypertension [31]. In our present study, we consistently found that both of $\mathrm{ABI}$ value and $\mathrm{ABI}<$ 0.9 also could predict overall and CV mortality in patients with AMI.

Furthermore, age was also shown to be a significant predictor for overall and CV mortality in our study. However, it was not difficult to understand that patients with older age may have higher overall and CV mortality.

\section{Study limitations}

There were some limitations to this study. First, the sample size of our study was not large, but the follow-up period was very long, up to 181 months. 
Second, we did not adjust hypertension medication in the multivariable analysis because of incomplete data. In our hospital, the chart of patient was not available if he or she did not visit our hospital again more than 10 years. Finally, although simultaneous 4 -limb blood pressure measurement was not difficult, many of cardiac or intensive care units lacked an adequate machine to perform such measurement.

\section{Conclusions}

In AMI patients, 4-limb blood pressure measurement could generate several useful parameters in predicting overall and CV mortality. Furthermore, ankle DBP on the lower side and interankle DBP difference were the most powerful parameters in prediction of overall and CV mortality, respectively. Hence, for better mortality prediction in AMI patients, simultaneous 4-limb blood pressure measurement was necessary and really had a big impact on the survival prediction.

\section{Acknowledgements}

\section{Mortality data were provided by the Collaboration Center of Health Information Application, Ministry of Health and Welfare, Executive Yuan. The research presented in this article is supported by the grants (KMUH103-3R06).}

\section{Competing Interests}

The authors have declared that no competing interest exists.

\section{References}

1. Verberk WJ, Kollias A, Stergiou GS. Automated oscillometric determination of the ankle-brachial index: a systematic review and meta-analysis. Hypertens Res. 2012; 35:883-891

2. Yu S, Zhou Y, Wu K, et al. Association of interarm blood pressure difference with cardio-cerebral vascular disease: A community-based, cross-sectional study. J Clin Hypertens (Greenwich). 2019:21:1115-1123.

3. Quiroga B, Galan I, Garcia de Vinuesa S, et al. Interarm systolic blood pressure as a predictor of cardiovascular events in patients with chronic kidney disease. Nephrol Dial Transplant. 2015; 30:801-806

4. Clark CE, Steele AM, Taylor RS, et al. Interarm blood pressure difference in people with diabetes: measurement and vascular and mortality implications: a cohort study. Diabetes Care. 2014; 37:1613-1620

5. Sheng CS, Liu M, Zeng WF, et al. Four-limb blood pressure as predictors of mortality in elderly Chinese. Hypertension. 2013; 61:1155-1160

6. Chen SC, Chang JM, Tsai YC, et al. Association of interleg BP difference with overall and cardiovascular mortality in hemodialysis. Clin J Am Soc Nephrol. 2012; 7:1646-1653

7. Lin JS, Olson CM, Johnson ES, et al. The ankle-brachial index for peripheral artery disease screening and cardiovascular disease prediction among asymptomatic adults: a systematic evidence review for the U.S. Preventive Services Task Force. Ann Intern Med. 2013; 159:333-341

8. Velescu A, Clara A, Marti R, et al. Abnormally High Ankle-Brachial Index is Associated with All-cause and Cardiovascular Mortality: The REGICOR Study. Eur J Vasc Endovasc Surg. 2017; 54:370-377

9. Samba H, Guerchet M, Ndamba-Bandzouzi B, et al. Ankle Brachial Index (ABI) predicts 2-year mortality risk among older adults in the Republic of Congo: The EPIDEMCA-FU study. Atherosclerosis. 2019; 286:121-127

10. Chen HY, Wei F, Wang LH, et al. Abnormal ankle-brachial index and risk of cardiovascular or all-cause mortality in patients with chronic kidney disease: a meta-analysis. J Nephrol. 2017; 30:493-501

11. Jones WS, Patel MR, Rockman CB, et al. Association of the ankle-brachial index with history of myocardial infarction and stroke. Am Heart J. 2014; 167:499-505
12. Attar R, Wester A, Koul S, et al. Peripheral artery disease and outcomes in patients with acute myocardial infarction. Open Heart. 2019; 6:e001004

13. Inglis SC, Bebchuk J, Al-Suhaim SA, et al. Peripheral artery disease and outcomes after myocardial infarction: an individual-patient meta-analysis of 28,771 patients in CAPRICORN, EPEHESUS, OPTIMAAL and VALIANT. Int J Cardiol. 2013; 168:1094-1101

14. Yamashina A, Tomiyama $\mathrm{H}$, Takeda $\mathrm{K}$, et al. Validity, reproducibility, and clinical significance of noninvasive brachial-ankle pulse wave velocity measurement. Hypertens Res. 2002; 25:359-364

15. Tomiyama $\mathrm{H}$, Yamashina $\mathrm{A}$, Arai $\mathrm{T}$, et al. Influences of age and gender on results of noninvasive brachial-ankle pulse wave velocity measurement--a survey of 12517 subjects. Atherosclerosis. 2003; 166:303-309

16. Martin U, Holder R, Hodgkinson J, et al. Inter-arm blood pressure differences compared with ambulatory monitoring: a manifestation of the 'white-coat' effect? Br J Gen Pract. 2013; 63:e97-103

17. Verberk WJ, Kessels AG, Thien T. Blood pressure measurement method and inter-arm differences: a meta-analysis. Am J Hypertens. 2011; 24:1201-1208

18. Clark CE, Aboyans V. Interarm blood pressure difference: more than an epiphenomenon. Nephrol Dial Transplant. 2015; 30:695-697

19. White J, Mortensen LH, Kivimaki M, et al. Interarm differences in systolic blood pressure and mortality among US army veterans: aetiological associations and risk prediction in the Vietnam Experience Study. Eur J Prev Cardiol. 2014; 21:1394-1400

20. Weinberg I, Gona P, O'Donnell CJ, et al. The systolic blood pressure difference between arms and cardiovascular disease in the Framingham Heart Study. Am J Med. 2014; 127:209-215

21. Kim J, Song TJ, Song D, et al. Interarm blood pressure difference and mortality in patients with acute ischemic stroke. Neurology. 2013; 80:1457-1464

22. Clark CE, Taylor RS, Shore AC, et al. Association of a difference in systolic blood pressure between arms with vascular disease and mortality: a systematic review and meta-analysis. Lancet. 2012; 379:905-914

23. Clark CE, Taylor RS, Shore AC, et al. The difference in blood pressure readings between arms and survival: primary care cohort study. BMJ. 2012; 344:e1327

24. Rahman F, Al Rifai M, Blaha MJ, et al. Relation of Diastolic Blood Pressure and Coronary Artery Calcium to Coronary Events and Outcomes (From the Multi-Ethnic Study of Atherosclerosis). Am J Cardiol. 2017; 120:1797-1803

25. Protogerou AD, Safar ME, Iaria $P$, et al. Diastolic blood pressure and mortality in the elderly with cardiovascular disease. Hypertension. 2007; 50:172-180

26. Chang Y, Kim J, Kim MH, et al. Interarm Blood Pressure Difference is Associated with Early Neurological Deterioration, Poor Short-Term Functional Outcome, and Mortality in Noncardioembolic Stroke Patients. J Clin Neurol. 2018; 14:555-565

27. Chen SC, Tsai YC, Huang JC, et al. Interankle systolic blood pressure difference and renal outcomes in patients with chronic kidney disease. Nephrology (Carlton). 2016; 21:379-386

28. Chen J, Mohler ER, Garimella PS, et al. Ankle Brachial Index and Subsequent Cardiovascular Disease Risk in Patients With Chronic Kidney Disease. J Am Heart Assoc. 2016;5:e003339.

29. Ono K, Tsuchida A, Kawai H, et al. Ankle-brachial blood pressure index predicts all-cause and cardiovascular mortality in hemodialysis patients. J Am Soc Nephrol. 2003; 14:1591-1598

30. Cardoso CRL, Melo JV, Salles GC, et al. Prognostic impact of the ankle-brachial index on the development of micro- and macrovascular complications in individuals with type 2 diabetes: the Rio de Janeiro Type 2 Diabetes Cohort Study. Diabetologia. 2018; 61:2266-2276

31. Luo YY, Li J, Xin Y, et al. Risk factors of peripheral arterial disease and relationship between low ankle brachial index and mortality from all-cause and cardiovascular disease in Chinese patients with hypertension. J Hum Hypertens. 2007; 21:461-466. 